\title{
The Complication Rate after Hypospadias Repair and Correlated Preoperative Symptoms*
}

\author{
Hans Winberg, Gunnar Westbacke, Ann Nozohoor Ekmark, Magnus Anderberg, \\ Einar Arnbjörnsson\# \\ Department of Pediatric Surgery, Skåne University Hospital and Institution of Clinical Research, \\ Lund University, Lund, Sweden \\ Email: "
}

Received 12 October 2014; revised 24 November 2014; accepted 20 December 2014

Academic Editor: Phillip Mucksavage, University of Pennsylvania, USA

Copyright (C) 2014 by authors and Scientific Research Publishing Inc.

This work is licensed under the Creative Commons Attribution International License (CC BY). http://creativecommons.org/licenses/by/4.0/

c) (7) Open Access

\section{Abstract}

Aim: To assess the rate of complications following hypospadias repair in a consecutive series of boys and the correlations of those complications with their preoperative symptoms, degree of hypospadias and method of operation. This study was conducted to address the question of whether all boys with all degrees of hypospadias should undergo reconstruction. Methods: This was a prospective cohort study. We included every boy who underwent an operation for the primary repair of hypospadias between January 2011 and April 2014. The median follow-up time was 24 months. The study ended in October 2014. The main outcome measurements were the frequency of postoperative complications and their correlations with the degree of hypospadias, the preoperative symptoms and the operative intervention performed. Results: Among the 76 boys who underwent operations, 23 had degree 1, 47 had degree 2, and 6 had degree 3 hypospadias. Preoperatively, 43 of the boys had symptoms that motivated the operation, including stenosis (38), a curvature (10) or both (5). Forty-three boys underwent operations with the MAVIS technique, 28 underwent TIP repair, 1 underwent a Duckett procedure, and 4 underwent Byar two-stage procedures. There were complications requiring reoperations including fistulas or ruptures in $26(34 \%)$ boys. There were no significant differences in the rates of complications with surgery, fistulas $(\mathrm{P}=$ $0.4775)$, ruptures $(P=0.2417)$ or other complications $(P=0.5165)$ between the groups with or without preoperative symptoms, those with different degrees of hypospadias or those who un-

\footnotetext{
"Part of the information in this manuscript has been presented as an abstract with the following number A-587-0025-00540 and an oral presentation with the title "Should all boys with hypospadias undergo reconstruction?" in the 4th World Congress of Pediatric Surgery (WOFAPS 2013) in 13-16 October 2013, in Berlin, Germany.

"Corresponding author.
}

How to cite this paper: Winberg, H., Westbacke, G., Ekmark, A.N., Anderberg, M. and Arnbjörnsson, E. (2014) The Complication Rate after Hypospadias Repair and Correlated Preoperative Symptoms. Open Journal of Urology, 4, 155-162. 
derwent different operative methods for repair. Conclusions: The complication rate in this series was high. The study was prospective, and no boy was lost during follow-up. Because the complication rate did not correlate with the degree of hypospadias nor the preoperative symptoms, there may be a group of boys with hypospadias without symptoms for whose operations are questionable. The preoperative symptoms should be reported in future reports of the results of hypospadias surgery.

\section{Keywords}

\section{Hypospadias Repair, Preoperative Symptoms, Age, Meatal Location, Outcome, Complications}

\section{Introduction}

Globally, hypospadias is one of the most common congenital anomalies [1]. The reconstruction for this condition is a delicate procedure, and based on the literature, it is clear that pediatric surgeons and plastic surgeons contribute to its understanding. The ideal method of repair for hypospadias is still under debate. More than 300 different operations have been described in the literature for the treatment of this condition [1]. This considerable number of different operative interventions might reflect the frustration of surgeons facing the high rate of complications that result from this type of surgery.

The call for comparisons of centers has grown stronger during recent decades, and the open presentation of data is mandatory to maintain humble attitudes and to refine hypospadias repair. Acknowledging this, we compared our results with those reported in the literature. The results are of importance for the preoperative information given to the guardians of boys with hypospadias. Furthermore, it is of interest to disclose whether postoperative complications are correlated with the degree of hypospadias, with the used for operative intervention, or with the boys' preoperative symptoms. In general, the symptoms of hypospadias depend on the degree of the condition. In very mild cases, the only drawback is cosmetic appearance. Spraying during urination is a common complaint. A downward directed stream can interfere with voiding in the standing position. In some cases, associated meatal stenosis has occurred and can result in an outlet obstruction. With increasing degrees of hypospadias, the risk of a curvature of the penis increases.

Guidelines or consensus in the literature regarding which boys with hypospadias should undergo reconstructions are typically vague and left to the judgment of the surgeon [2]. A validated scheme for the evaluation of hypospadias repair was missing in the literature until the report on the hypospadias objective scoring evaluation (HOSE) system score was published [3]. Information from a scoring system like this could, if used globally, be important for comparing the outcomes of boys who have undergone operations at different centers and for the preoperative information provided to the guardians of boys with hypospadias. However, we still lack an instrument to evaluate boys with hypospadias who do not undergo surgery. We are not aware of any similar prospective studies that have aimed to search for information that can be used to select those who should not undergo hypospadias operations.

\section{Patients and Methods}

\subsection{Ethical Consideration}

The study protocol was designed to meet the legislative documentation required in the country of origin. The intention to treat was the main analysis strategy and was applied to all boys. The regional research ethics committee approved the study. The authors have no disclosures.

\subsection{Patients}

All of the boys with different degrees of hypospadias were referred to a tertiary center of pediatric surgery and were prospectively registered from January 2011 to April 2014. All primary urethra reconstructions during this period were included. The senior hypospadias surgeon at the hospital or a surgeon tutored by him performed all of the reconstructions. 


\subsection{Methods}

The hypospadias were classified according to the preoperative position of the urethral meatus as follows [4]:

- Degree 1: glandular hypospadias with the meatus on the glans or in the sulcus coronaries,

- Degree 2: distal hypospadias with the meatus subcoronally or on the mid-shaft of the penis,

- Degree 3: with the meatus penoscrotally or scrotally,

- In cases where a curvature was present, the classification of the hypospadias was made after the straightening procedure.

Four different operative techniques were used, and all techniques are well-established in the literature [4]. The technique chosen for each boy depended on the degree of hypospadias as described above, the individual prerequisite and the judgment of the surgeon. The Mathieu and "V" incision sutured meatoplasty (MAVIS) [5] [6] was used for all degrees of hypospadias, whereas the tabularized incised plate repair (TIP) [7] was used for degrees 1 and 2. A Duckett reconstruction [8] was performed in one degree 2 patient, and in the two most pronounced degree 3 boys were used the Byar two-stage reconstruction [9].

\subsection{Catheters}

For the TIP repairs, 6- or 8-French (F) stents that dripped into the nappy were retained for seven days. When a MAVIS or a Duckett plasty was performed, a suprapubic catheter 10 Charrier (Ch) was used for seven days in all cases. For the tabularization of the neourethra in the Duckett plasties, conventional Foley ${ }^{\mathrm{TM}} \mathrm{Ch} 8$ or 10 catheters were used.

\subsection{Suture Technique}

For the urethral reconstruction, 5/0 or 6/0 polyglactin (Vicryl ${ }^{\mathrm{TM}}$ ) and polydioxanone (PDS used. They were sewn either with running or interrupted patterns according to the choice of the surgeon based on the local prerequisites.

\subsection{Dressings}

The so-called chimney dressing used was the same for the patients in the MAVIS, Duckett and Byar I-II groups. This dressing consisted of a layer of Mepitel ${ }^{\mathrm{TM}}$, which was a transparent, flexible thin perforated polyurethane film embedded in Safetac ${ }^{\mathrm{TM}}$ (a silicon wound contact layer), a layer of compressing gauze and a surrounding tape. These dressings resembled a chimney. The dressing used for the TIP operations consists of a piece of Aquacel $^{\mathrm{TM}}$ covering the suture line, Tegaderm ${ }^{\mathrm{TM}}$ wrapping, compression gauze bandage, PEHA Haft ${ }^{\mathrm{TM}}$ and fluffed gauze compresses. The dressings were all removed under general anesthesia one week after the procedure.

\subsection{Antibiotics}

Prior to all procedures, one dose of preoperative prophylactic sulphamethoxazole and trimethoprim was given intravenously to all of the patients.

\subsection{Postoperative Regime}

The boys who underwent MAVIS and TIP repair operations were discharged on day three after the operations. Their parents were instructed to keep the child in a supine or sitting position during the first week. When a Duckett plasty or a Byars II-reconstruction was performed, the boys were hospitalized and immobilized in a wheelchair for a week.

\subsection{Secondary Corrections}

In cases in which a TIP reconstruction was made, the parents might have requested a preputial plasty or a circumcision, either of which could be performed in the same session [10]. For the MAVIS procedure, we preferred to do these corrections in a secondary setting.

\subsection{Clinical Follow-Up}

The patients were seen in a week after the reconstruction when the catheter and dressing were removed. They 
were then called back to the out-patient clinic at three weeks and two and six months after the operation. In cases in which no complications occurred, a final clinical examination was performed in one year after the reconstruction. During the visits, the patients were examined regarding urinary function including urinary flow measurements when applicable; i.e., if the patient was old enough and cooperative. The examinations of the surgical results focused on function, cosmetic appearance and complications such as fistulas, strictures, ruptures, postoperative infections, haematomas or bleeding, malfunctioning of catheters, and urinary retention. Moreover, we registered the techniques used and the age at the time of the urethral reconstruction.

\subsection{Statistical Analysis}

Data were analyzed by Fisher's exact probability test, two tailed, as well as non-parametric tests including the Mann-Whitney U test. A P-value less than 0.05 was considered significant.

\section{Results}

Seventy-six boys underwent primary hypospadias repair during the 40-month period. Their median age (range) at the time of repair was $3(0-8)$ years, and the median (range) follow-up period was $19(1-40)$ months. No boy was lost during follow-up.

The preoperative statuses and evaluations of function are summarized in Table 1. Ten of the patients were described as having curvatures that might have interfered with future sexual function and thus motivated the operations. Thirty-eight boys had meatal stenosis. Nineteen had meatotomias performed prior to the urethral reconstructions, and another 19 had the meatotomias performed as a part of the urethral reconstruction. Uroflowmetry examinations were performed in 30 boys.

The stenosis is defined as difficulty urinating. The boys may have urinary frequency, prolonged urination, a thin stream, and difficulty starting urinary flow, keeping the flow going, difficulty in aiming the stream into the toilet, spraying of the urinary stream, and straining or arching of the back during voiding. These clinical findings may be supported by uroflowmetry measuring the volume, the speed with which it is released and duration of urine released from the body. Furthermore, bladder ultrasound can be used to detect urinary retention.

The most preferred method of reconstruction was the MAVIS, which was applied for any degree of hypospadias and in 13, 27 and 3 boys with degrees 1, 2 and 3, respectively. The TIP method was used for degree 1 in 10 boys and for mild degree 2 in 18 boys.

The results of the study are summarized in Tables 2-4.

- Table 2 summarizes the postoperative complications that were correlated with the meatal location. The numbers of complications did not differ significantly between the groups with different degrees of hypospadias.

- Table 3 shows the methods used for the operations. In the MAVIS group, 18 (41\%) had a fistula or rupture that necessitated reoperation. The corresponding number for the TIP procedures was 4 (15\%), which was significantly lower, and the Byar 2 stage and Duckett plasties all required further surgery.

Table 1. Preoperative clinical evaluations of the 76 boys with Hypospadias. Their median age (range) at the time of repair was $3(0$ - 8) years, and the median (range) follow-up period was $19(1$ - 40) months.

\begin{tabular}{|cccc}
\hline \multicolumn{2}{c}{ Anatomical malformation } & \multicolumn{2}{c}{ Penis function } \\
\hline Ventral curvature ( $\mathrm{n}=)$ & Grade of hypospadias ( $\mathrm{n}=)$ & Expected urination problem in $(\mathrm{n}=)$ & Expected copulation problem in ( $\mathrm{n}=)$ \\
\hline - Yes (10) & I (1) & 1 & 1 \\
& II (6) & 4 & 3 \\
III (3) & I (22) & 12 & \\
& II (41) & 20 \\
Meatal stenosis (38) & III (3) & 1 \\
\hline
\end{tabular}


Table 2. The complications of hypospadias including fistulas, rupture and others complications and their correlations with the meatal locations described using the different degrees of hypospadias.

\begin{tabular}{lcccc}
\hline Degree of hypospadias & 1 & 2 & 3 & P-value* $^{*}$ \\
\hline - $\mathrm{n}=$ & 23 & 47 & 6 & \\
\hline Complications & & & & 0.4775 \\
\hline - Fistulas & 5 & 14 & 3 & 0.2417 \\
- Ruptures & 2 & 1 & 1 & 0.5615 \\
- Others & 2 & 9 & 0 & \\
\hline
\end{tabular}

"The Freeman-Halton extension of Fisher's exact test was used to compute the (two-tailed) probability of obtaining the distribution of values in a $2 \times$ 3 contingency table given the number of observations in each cell.

Table 3. The method of operation correlated with the postoperative complications ${ }^{*}$. The included boys were grouped in to 4 groups according to the method used for the reconstruction surgeries. However, the case numbers of two of the groups were very small and resulted in statistical instability. Therefore, the patients were merged into 3 groups.

\begin{tabular}{cccccc}
\hline & Total & TIP $(\mathrm{n}=28)$ & MAVIS (n=43) & $\begin{array}{c}\text { Dockets ( } \mathrm{n}=1) \\
\text { Byar (n=4) }\end{array}$ & P-value $^{* *}$ \\
\hline Fistulas & $22(29 \%)$ & $3(11 \%)$ & $15(35 \%)$ & $4(80 \%)$ & 0.0216 \\
Rupture & $4(5 \%)$ & $1(4 \%)$ & $3(7 \%)$ & & 0.2815 \\
Meatal stenosis & $1(1 \%)$ & $1(4 \%)$ & 0 & 0 & 0.3803 \\
Infections & $2(3 \%)$ & 0 & $2(5 \%)$ & 0 & 0.3807 \\
Hematoma/bleeding & $4(5 \%)$ & $3(11 \%)$ & $1(2 \%)$ & 0 & 0.1325 \\
Catheter malfunction & $2(3 \%)$ & $2(7 \%)$ & 0 & $1(20 \%)$ & 0.1413 \\
Urinary retention & $1(1 \%)$ & 0 & 0 & 0.0111 & 1.0 \\
P-value & & 0.1649 & 0.2086 & 0 \\
\hline
\end{tabular}

*Glans dehiscence, urethral stricture or diverticulum were not found; ${ }^{* *}$ The Fisher's exact test was used to compute the (two-tailed) probability of obtaining the distribution of values in a $2 \times 2$ contingency table given the number of observations in each cell; ${ }^{* * *}$ Mann-Whitney U Test (two-tailed). The statistical significance found for the comparison of the Duckett and Byar procedures was considered to be of no clinical significance due to the small number of boys.

Table 4. Clinical symptoms and postoperative complications in 76 consecutive patients who underwent primary reconstruction for hypospadias repair.

\begin{tabular}{lcccc}
\hline Clinical symptoms & Curvature & Stenosis & Curvature and stenosis & P-value $^{*}$ \\
\hline$-\mathrm{n}=$ & 10 & 38 & 5 & \\
\hline Complications & & & & 0.3994 \\
\hline - Fistulas & $5(50 \%)$ & $10(26 \%)$ & 0 & 1.0 \\
- Ruptures & 0 & $0(0 \%)$ & $1(20 \%)$ & 0.8536 \\
\hline - Others & $1(10 \%)$ & $9(24 \%)$ & 0 \\
\hline
\end{tabular}

*The Freeman-Halton extension of Fisher's exact test was used to compute the (two-tailed) probability of obtaining the distribution of values in a $2 \times$ 3 contingency table given the number of observations in each cell.

- The clinical symptoms that preceded the repairs and were correlated with the postoperative complications are shown in Table 4, and there were no differences regarding the postoperative complications.

\section{Discussion}

In our study, we noted complications that were insignificantly different from those that have previously been described in comparable reports collected from the literature the last few years $(P=0.0547)$ [11]-[16], Table 5. 
Table 5. Summary of reports found in the literature published during the period 2008-2013.

\begin{tabular}{ccccc}
\hline Author & Year of publication & Number of boys TIP/MAVIS & Number of complications (\%) & Re-do op (\%) \\
\hline Aslam [10] & 2013 & 74 & $5(7)$ & $5(7)$ \\
Spinoit [11] & 2013 & 474 & $114(24.1)$ & $114(24.1)$ \\
Prat [12] & 2012 & 271 & $101(37.3)$ & $13(11.8)$ \\
Elganainy [13] & 2010 & 110 & $11(13.8)$ \\
Sarhan [14] & 2009 & 80 & $5(12.5)$ \\
Aminsharifi [15] & 2008 & 40 & $249(23.7)$ \\
Summary & & 1049 & \\
\hline
\end{tabular}

However, there are reports that have described results that are significantly worse than ours [13] $(P=0.0327)$. The reasons for the high frequency of complications are probably multifactorial and include the following:

- The type of study and compliance with the follow-ups; our case was a prospective study and we did not lose any boy to follow-up.

- The interpretation of the degree of the hypospadias might have differed between different surgeons. The group of boys described in this study was evaluated based on the consensus between the two operating surgeons.

- The age at reconstruction is the subject of debate [1]. In this study, the age of the boys at operation was uniform.

- The operative intervention chosen and the interpretation of the choice should be discussed because more than 300 different operative interventions have been described in the literature [1].

- The operating surgeon. In this series, two experienced and dedicated surgeons operated on the boys. The older surgeon has more than 20 years of experience with hypospadias surgeries and over 30 years as a paediatric surgeon. The younger surgeon has six years of experience with hypospadias surgery and more than 15 years in general and paediatric surgery.

- The suturing material and the suturing technique should be further studied. Currently, no consensus regarding the sutures or suture techniques is available in the literature [1].

- The use of prophylactic antibiotics. Consensus is missing, and guidelines are not available [1].

- The catheters and stents used and their route differs between different centres.

- The dressings, mobilization plan, etc. are different from between centres and vary from day-case fast-track surgery to in-hospital durations of 3 - 5 days [1].

The outcome measurements in this study included the frequencies of postoperative complications that were correlated with preoperative symptoms, the degree of hypospadias (i.e., the meatal location) and the various operative interventions. The results can only explain whether there were differences between the different parameters studied and cannot indicate differences between operations and non-operations because all of the boys underwent operative interventions. Thus, the result indirectly add information to the research question of whether all boys with hypospadias should undergo reconstruction by pointing out that unnecessary operations on boys without symptoms cause complication with a frequency that is similar to those for whose operative interventions are necessary.

The strength of this study is that it is a prospective study and that only two surgeons operated on the boys. Moreover, all of the boys were present at follow-up.

The weaknesses of this study are that the population was small, and although effort was expended to define the degrees of hypospadias, bias in the interpretations of those degrees must be taken into account. Underestimations of the degrees due to insidious curvatures might have occurred. The operating surgeons were also responsible for the follow-up, which might have caused a conflict of interest. Furthermore, the non-randomized design of the study is a limitation.

A median age of 3 years is certainly older than average of boys operated on for hypospadias. Various studies have shown an increased incidence of complications with later age at surgery. Therefore, the majority of pediatric urologists would now perform hypospadias repairs at about one year of age. It is therefore unlikely that the 
patients ever have symptoms and signs such as curvature or stenosis which may be invisible at an early stage.

One may have concerns that the wrong operations have been performed for the more proximal hypospadias and those with significant curvature, and that this is expressed by the high, 50\%, fistula rate in boys with curvature. These boys should probably not have had a single stage repair. It may be a bold endeavor to perform single stage repairs on grade 3 hypospadias in the presence of a significant intrinsic chordee [17].

An indication for a suprapubic catheter in the boys included in this study can be discussed. The vast majority of pediatric urologists would perform this surgery as day cases or at the most an overnight stay. There is no need for immobilization or a wheelchair. A critical review of the practice is performed with the intention to benchmark it with current pediatric urological practice.

Small boys seldom have any subjective problems from their hypospadias. So the mean objective for surgical treatment in childhood is basically to prevent problems later in life. It is hard to understand how a conclusion can be drawn in respect to overall function, voiding and erection in adult life, which, according to today's perspective by most authors, is the age of final judgments.

A question of interest is why the intermediate group of hypospadias, Type 2, is a larger group than the milder. As far as we are aware that there has been no selection of patients as they are included consecutively as they are referred to the hospital and there are no exclusions.

There was no correlation between preoperatively symptoms, grade of hypospadias or operative technique. Thus, in this material it was not possible to tell which patients would suffer from a postoperative complication by using the indicated preoperative parameters.

The findings call for a discussion of whether the boys with low-grade hypospadias should undergo reconstructive surgery. There might be some boys with mild hypospadias that would do better without an operation because of the risk of complications do not seem to correlate with the degree of hypospadias and the complications that might be discovered as late as at post pubertal examinations [18]. In our study, there was a significantly increased risk associated with degree 3 hypospadias, and this finding is in line with those of earlier studies; however, the number of boys in this group was too small to reach a conclusion. A surgical intervention for a penis that can function normally without any operative intervention that leaves the boy with unnecessary scars on the penis should be avoided. If the indication for operation is meatal stenosis, this condition can be treated with a meatotomia only. Then, if the meatus is sufficiently wide (operated on or not) and the curvature neither inhibits growth nor function, the only reason to perform an operation would be the non-acceptance of the aesthetic appearance. Is it possible to develop criteria for an acceptable location of the meatus or for the looks of the preputium? How high a complication rate should the surgeon and the patient/guardian be prepared to tolerate for an operation that primarily addresses an aesthetic issue? How important should we allow genital aesthetics to be? Any decisions regarding solely cosmetic intervention should be left to the boy when he is old enough to make his own decisions.

\section{Conclusion}

This prospective study revealed that the complication rate following hypospadias surgery did not correlate with the preoperative symptoms or the degree of hypospadias. Thus, there may be a group of boys with hypospadias without symptoms for whose operations are questionable. The preoperative symptoms should be reported in future reports on the results of hypospadias surgery.

\section{Conflict of Interest Statement}

All authors disclose no financial or personal relationships with other people or organizations that could inappropriately influence (bias) their work. This includes employment, consultancies, stock ownership, honoraria, paid expert testimony, patent applications/registrations, and grants or other funding. There were no fundings.

\section{References}

[1] Cimador, M., Vallasciani, S., Manzoni, G., Rigamonti, W., De Grazia, E. and Castagnetti, M. (2013) Failed Hypospadias in Paediatric Patients. Nature Reviews Urology, 10, 657-666. http://dx.doi.org/10.1038/nrurol.2013.164

[2] Tekgül, S., Riedmiller, H., Gerharz, E., Hoebeke, P., Kocvara, R., Nijman, R., et al. (2009) Guidelines on Paediatric Urology. European Association of Urology, European Society for Paediatric Urology, Arnhem, The Netherlands, 18-22. 
[3] Holland, A.J., Smith, G.H., Ross, F.I. and Cass, D.T. (2001) HOSE: An Objective Scoring System for Evaluating the Results of Hypospadias Surgery. BJU International, 88, 255-258. http://dx.doi.org/10.1046/j.1464-410x.2001.02280.x

[4] Hadidi, A. (2004) Classification of Hypospadias. In: Hadidi, A. and Azmy, A., Eds., Hypospadias Surgery. An Illustrated Guide, Springer Verlag, Berlin, 79-82.

[5] Mathieu, P. (1932) Traitement en un temps de l'hypospade balanique et juxta-balanique. Journal de Chirurgie (Paris), 39, 481-484.

[6] Boddy, S.A. and Samuel, M. (2000) Mathieu and "V” Incision Sutured (MAVIS) Results in a Natural Glanular Meatus. Journal of Pediatric Surgery, 35, 494-496. http://dx.doi.org/10.1016/S0022-3468(00)90220-3

[7] Snodgrass, W.T. (1999) Tubularized Incised Plate Hypospadias Repair: Indications, Technique, and Complications. Urology, 54, 6-11. http://dx.doi.org/10.1016/S0090-4295(99)00144-2

[8] Duckett Jr., J.W. (1980) Transverse Preputial Island Flap Technique for Repair of Severe Hypospadias. The Urologic Clinics of North America, 7, 423-430.

[9] Byars, L.T. (1955) A Technique for Consistently Satisfactory Repair of Hypospadias. Surgery, Gynecology and Obstetrics, 100, 184-190.

[10] Snodgrass, W.T., Koyle, M.A., Baskin, L.S. and Caldamone, A.A. (2006) Foreskin Preservation in Penile Surgery. The Journal of Urology, 176, 711-714. http://dx.doi.org/10.1016/j.juro.2006.03.082

[11] Aslam, R., Campbell, K., Wharton, S. and Bracka, A. (2013) Medium to Long Term Results Following Single Stage Snodgrass Hypospadias Repair. Journal of Plastic Reconstructive and Aesthetic Surgery, 66, 1591-1595. http://dx.doi.org/10.1016/j.bjps.2013.06.041

[12] Spinoit, A.F., Poelaert, F., Groen, L.A., Van Laecke, E. and Hoebeke, P. (2013) Hypospadias Repair at a Tertiary Care Center: Long-Term Follow-Up Is Mandatory to Determine the Real Complication Rate. Journal of Urology, 189, 2276-2281. http://dx.doi.org/10.1016/j.juro.2012.12.100

[13] Prat, D., Natasha, A., Polak, A., Koulikov, D., Prat, O., Zilberman, M., et al. (2012) Surgical Outcome of Different Types of Primary Hypospadias Repair during Three Decades in a Single Center. Urology, 79, 1350-1353. http://dx.doi.org/10.1016/j.urology.2011.11.085

[14] Elganainy, E.O., Abdelsalam, Y.M., Gadelmoula, M.M. and Shalaby, M.M. (2010) Combined Mathieu and Snodgrass Urethroplasty for Hypospadias Repair: A Prospective Randomized Study. International Journal of Urology, 17, 661665. http://dx.doi.org/10.1111/j.1442-2042.2010.02537.x

[15] Sarhan, O., Saad, M., Helmy, T. and Hafez, A. (2009) Effect of Suturing Technique and Urethral Plate Characteristics on Complication Rate Following Hypospadias Repair: A Prospective Randomized Study. Journal of Urology, 182, 682-685. http://dx.doi.org/10.1016/j.juro.2009.04.034

[16] Aminsharifi, A., Taddayun, A., Assadolahpoor, A. and Khezri, A. (2008) Combined Use of Mathieu Procedure with Plate Incision for Hypospadias Repair: A Randomized Clinical Trial. Urology, 72, 305-308. http://dx.doi.org/10.1016/j.urology.2008.02.034

[17] Joseph, V.T. (2003) One-Stage Surgical Correction of Proximal Hypospadias. Annals of the Academy of Medicine, Singapore, 32, 106-111.

[18] Ekmark, A.N., Svensson, H., Arnbjörnsson, E. and Hansson, E. (2013) Postpubertal Examination after Hypospadias Repair Is Necessary to Evaluate the Success of the Primary Reconstruction. European Journal of Pediatric Surgery, 23, 304-311. http://dx.doi.org/10.1055/s-0033-1333638 
Scientific Research Publishing (SCIRP) is one of the largest Open Access journal publishers. It is currently publishing more than 200 open access, online, peer-reviewed journals covering a wide range of academic disciplines. SCIRP serves the worldwide academic communities and contributes to the progress and application of science with its publication.

Other selected journals from SCIRP are listed as below. Submit your manuscript to us via either submit@scirp.org or Online Submission Portal.
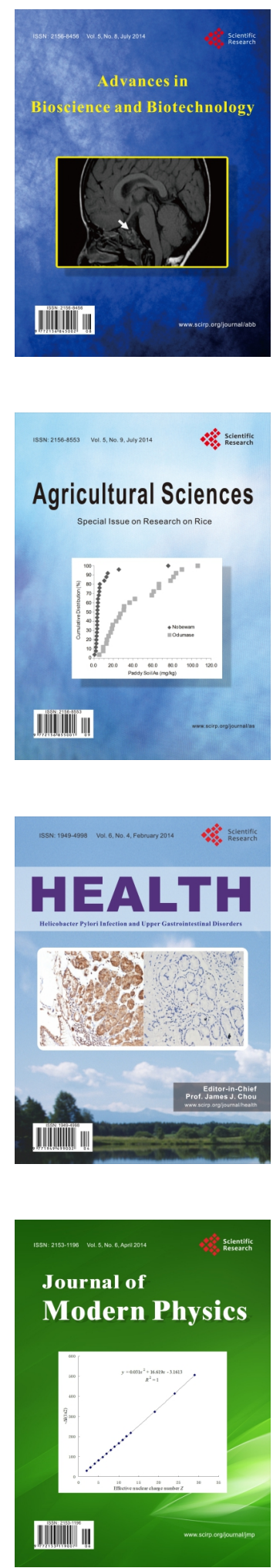
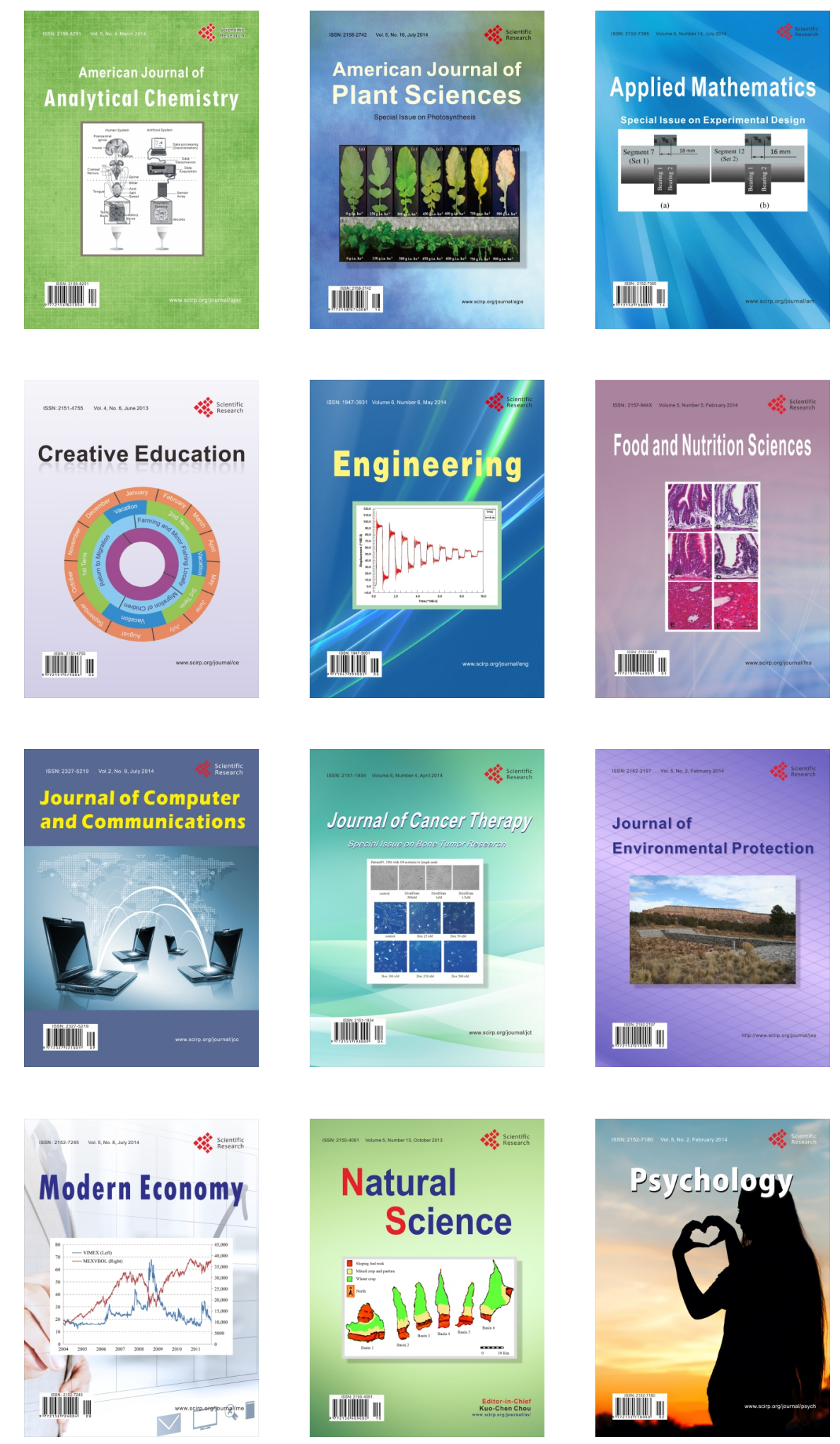